\title{
On maximizing a monotone $k$-submodular function under a knapsack constraint
}

\author{
Zhongzheng Tang ${ }^{1}$ Chenhao Wang ${ }^{2}$ Hau Chan $^{2}$ \\ 1 School of Science, Beijing University of Posts and Telecommunications, \\ Beijing, China \\ 2 Department of Computer Science and Engineering, University of Nebraska-Lincoln, \\ Lincoln, NE, United States \\ tangzhongzheng@amss.ac.cn, \{cwang55,hchan3\}@unl.edu
}

\begin{abstract}
We study the problem of maximizing a monotone $k$-submodular function $f$ under a knapsack constraint, where a $k$-submodular function is a natural generalization of a submodular function to $k$ dimensions. We present a deterministic $\left(\frac{1}{2}-\frac{1}{2 e}\right)$-approximation algorithm that evaluates $f O\left(n^{5} k^{4}\right)$ times.
\end{abstract}

\section{Introduction}

A $k$-submodular function is a generalization of submodular function, where the input consists of $k$ disjoint subsets of the domain, instead of a single subset. The $k$-submodular maximization problem appears in a broad range of applications (e.g., influence maximization with $k$ kinds of topics and sensor placement with $k$ kinds of sensors [6]), due to the property of diminishing returns. The $k$-submodular maximization has been studied in the unconstrained setting [11], under cardinality constraints [6], and under matroid constraints [8]. In this note, we study the maximization problem of a nonnegative monotone $k$-submodular function under a knapsack constraint.

Let $V$ be a finite set. Let $(k+1)^{V}:=\left\{\left(X_{1}, \ldots, X_{k}\right) \mid X_{i} \subseteq V \forall i \in[k], X_{i} \cap X_{j}=\emptyset \forall i \neq j\right\}$ be the family of $k$ disjoint sets, where $[k]:=\{1, \ldots, k\}$. A function $f:(k+1)^{V} \rightarrow \mathbb{R}$ is called $k$-submodular [3], if for any $\mathbf{x}=\left(X_{1}, \ldots, X_{k}\right)$ and $\mathbf{y}=\left(Y_{1}, \ldots, Y_{k}\right)$ in $(k+1)^{V}$, we have

$$
f(\mathbf{x})+f(\mathbf{y}) \geq f(\mathbf{x} \sqcup \mathbf{y})+f(\mathbf{x} \sqcap \mathbf{y}),
$$

where

$$
\begin{aligned}
& \mathbf{x} \sqcup \mathbf{y}:=\left(X_{1} \cup Y_{1} \backslash\left(\bigcup_{i \neq 1} X_{i} \cup Y_{i}\right), \ldots, X_{k} \cup Y_{k} \backslash\left(\bigcup_{i \neq k} X_{i} \cup Y_{i}\right)\right), \\
& \mathbf{x} \sqcap \mathbf{y}:=\left(X_{1} \cap Y_{1}, \ldots, X_{k} \cap Y_{k}\right) \text {. }
\end{aligned}
$$


Denote $\mathbf{x} \preceq \mathbf{y}$, if $\mathbf{x}=\left(X_{1}, \ldots, X_{k}\right)$ and $\mathbf{y}=\left(Y_{1}, \ldots, Y_{k}\right)$ with $X_{i} \subseteq Y_{i}$ for each $i \in[k]$. For $\mathbf{x} \preceq \mathbf{y}$, define $\mathbf{y} \backslash \mathbf{x}=\left\{a \in V \mid a \in Y_{i} \backslash X_{i}, \forall i \in[k]\right\}$. Define the marginal gain when adding item $a$ to the $i$-th dimension of $\mathbf{x}$ to be

$$
\Delta_{a, i}(\mathbf{x}):=f\left(X_{1}, \ldots, X_{i-1}, X_{i} \cup\{a\}, X_{i+1}, \ldots, X_{k}\right)-f(\mathbf{x}) .
$$

It is not hard to see that, a $k$-submodular function $f$ satisfies orthant submodularity, that is,

$$
\Delta_{a, i} f(\mathbf{x}) \geq \Delta_{a, i} f(\mathbf{y}), \text { for any } \mathbf{x}, \mathbf{y} \in(k+1)^{V} \text { with } \mathbf{x} \preceq \mathbf{y}, a \notin \cup_{j \in[k]} Y_{j}, i \in[k] .
$$

A function $f:(k+1)^{V} \rightarrow \mathbb{R}$ is called monotone, if $f(\mathbf{x}) \leq f(\mathbf{y})$ for any $\mathbf{x} \preceq \mathbf{y}$. Ward and Živnỳ [11] shows that when monotonicity holds, $f$ is $k$-submodular if and only if it is orthant submodular.

Related works. It is well known that the diminishing return property characterizes the submodular function. This property often appears in practice, and so various problems can be formulated as submodular function maximization. Unfortunately, submodular function maximization is known to be NP-hard. Therefore, approximation algorithms that can run in polynomial time have been extensively studied for submodular function maximization, some of which consider various constraints. One decade ago, Huber and Kolmogorov [3] proposed $k$-submodular functions, which express the submodularity on choosing $k$ disjoint sets of elements instead of a single set, and recently become a popular subject of research [1, 2, 5, 9].

For the unconstrained non-monotone $k$-submodular maximization, Ward and Živnỳ [11 proposed a $\max \left\{\frac{1}{3}, \frac{1}{1+a}\right\}$-approximation algorithm with $a=\max \left\{1, \sqrt{\frac{k-1}{4}}\right\}$. Later, Iwata et al. [4] improved the approximation ratio to $\frac{1}{2}$, which is improved to $\frac{k^{2}+1}{2 k^{2}+1}$ by Oshima [7] more recently. For unconstrained monotone $k$-submodular maximization, Ward and Živnỳ [11] proven that a greedy algorithm is $\frac{1}{2}$-approximaion, and later, Iwata et al. [4] proposed a randomized $\frac{k}{2 k-1}$-approximation algorithm, which is asymptotically tight.

In the constrained setting, Ohsaka and Yoshida [6] proposed a $\frac{1}{2}$-approximation algorithm for nonnegative monotone $k$-submodular maximization with a total size constraint (i.e., $\cup_{i \in[k]}\left|X_{i}\right| \leq$ $B$ for an integer $B$ ) and a $\frac{1}{3}$-approximation algorithm for that with individual size constraints (i.e., $\left|X_{i}\right| \leq B_{i} \forall i \in[k]$ with integers $B_{i}$ ). Sakaue [8] proposed a $\frac{1}{2}$-approximation algorithm for nonnegative monotone $k$-submodular maximization with a matroid constraint. Thus, our work completes the picture by studying a knapsack constraint.

\section{Preliminaries}

For notational ease, we identify $(k+1)^{V}$ with the set

$$
\mathscr{S}=\left\{\cup_{j=1}^{t}\left\{\left(a_{j}, i_{j}\right)\right\} \mid a_{j} \in V i_{j} \in[k] \forall j \in[t], a_{j} \neq a_{j^{\prime}} \forall j \neq j^{\prime}\right\},
$$

that is, any $k$-disjoint set $\mathbf{x}=\left(X_{1}, \ldots, X_{k}\right) \in(k+1)^{V}$ uniquely corresponds to an item-index pairs set $S \in \mathscr{S}$, such that $\left(a_{j}, i_{j}\right) \in S$ if and only if $a_{j} \in X_{i_{j}}$. From now on, we rewrite $f(\mathbf{x})$ as $f(S)$ with some abuse of notations, and thus $\Delta_{a, i}(S)$ means the marginal gain $f(S \cup\{(a, i)\})-f(S)$. For any $S \in \mathscr{S}$, we define $U(S):=\{a \in V \mid \exists i \in[k]$ s.t. $(a, i) \in S\}$ to be the set of items included, and the size of $S$ is $|S|=|U(S)|$. In the remainder of this note, let $f$ be an arbitrary non-negative, 
monotone, $k$-submodular function. We further assume that $f(\emptyset)=0$, which is without loss of generality because otherwise we can redefine $f(S):=f(S)-f(\emptyset)$ for all $S \in \mathscr{S}$.

We first introduce an important lemma.

Lemma 2.1. For any $S, S^{\prime} \in \mathscr{S}$ with $S \subseteq S^{\prime}$, we have

$$
f\left(S^{\prime}\right)-f(S) \leq \sum_{(a, i) \in S^{\prime} \backslash S} \Delta_{a, i}(S)
$$

Proof. Let $t=\left|S^{\prime}\right|-|S|$. Define arbitrary manner sets $\left\{S_{j}\right\}_{j=0}^{t}$ such that (1) $S_{0}=S ;(2)\left|S_{j} \backslash S_{j-1}\right|=$ 1 for $j \in[t]$; (3) $S_{t}=S^{\prime}$. Let $\left\{\left(a_{j}, i_{j}\right)\right\}:=S_{j} \backslash S_{j-1}$ for $j \in[t]$. Then we have

$$
f\left(S^{\prime}\right)-f(S)=\sum_{j=1}^{t} \Delta_{a_{j}, i_{j}}\left(S_{j-1}\right) \leq \sum_{j=1}^{t} \Delta_{a_{j}, i_{j}}(S),
$$

where the inequality follows from the orthant submodularity.

The following theorem from Ward and Živnỳ [11] says that Greedy (see Algorithm 11) is $\frac{1}{2}$ approximation for maximizing $f$ without constraint (note that Theorem 5.1 of [11] states a more general conclusion which holds for a large class of $k$-set functions). Greedy considers items in an arbitrary order, and assign each item the best index that brings the largest marginal gain.

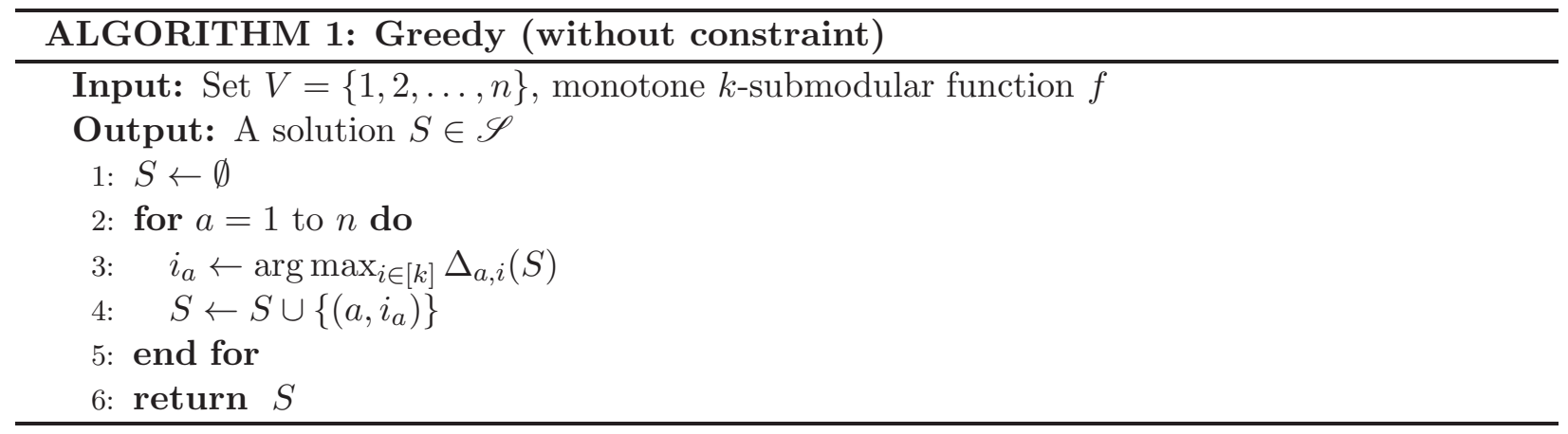

Theorem 2.2 ([11]). Let $T \in \mathscr{S}$ be a solution that maximizes $f$ in the unconstrained setting, and $S \in \mathscr{S}$ be the solution returned by Greedy. Then $f(T) \leq 2 \cdot f(S)$.

In the later proofs we will use the following inequality from Wolsey [12.

Lemma 2.3 ([12]). If $P$ and $D$ are arbitrary positive integers, $\left(\rho_{i}\right)_{i=1}^{P}$ are arbitrary nonnegative reals with $\rho_{1}>0$, then

$$
\frac{\sum_{i=1}^{P} \rho_{i}}{\min _{t=1, \ldots, P}\left(\sum_{i=1}^{t-1} \rho_{i}+D \rho_{t}\right)} \geq 1-\left(1-\frac{1}{D}\right)^{P} \geq 1-e^{-P / D} .
$$

\section{Knapsack constraint}

Given set $V=\{1, \ldots, n\}$, nonnegative integers $c_{a} \in \mathbb{R}$ for all $a \in V$, and budget $B \in \mathbb{R}$, we consider the following maximization problem with a knapsack constraint,

$$
\max _{S \in \mathscr{S}}\left\{f(S): \sum_{a \in U(S)} c_{a} \leq B\right\} \text {. }
$$


Sviridenko [10] considers the special case of $k=1$ (i.e., submodular maximization with a knapsack constraint), and presents a greedy $\left(1-\frac{1}{e}\right)$-approximation algorithm. We adapted it to Algorithm 2 for problem (11), and prove an approximation ratio of $\frac{1}{2}-\frac{1}{2 e}$. For any solution $S \in \mathscr{S}$, define $c(S)$ to be the total cost of all items in $S$.

In line 1 of Algorithm 2, it enumerates all feasible solutions of size one or two, and store the currently best solution as $S_{A}$; it takes $O\left(n^{2} k^{2}\right)$ oracle queries. Then it considers all feasible sets of size three, and completes each such set greedily with respect to the density, subject to the knapsack constraint. There are $O\left(n^{3} k^{3}\right)$ such sets, and for each set it takes $O\left(n^{2} k\right)$ queries. Thus, the time complexity is $O\left(n^{5} k^{4}\right)$.

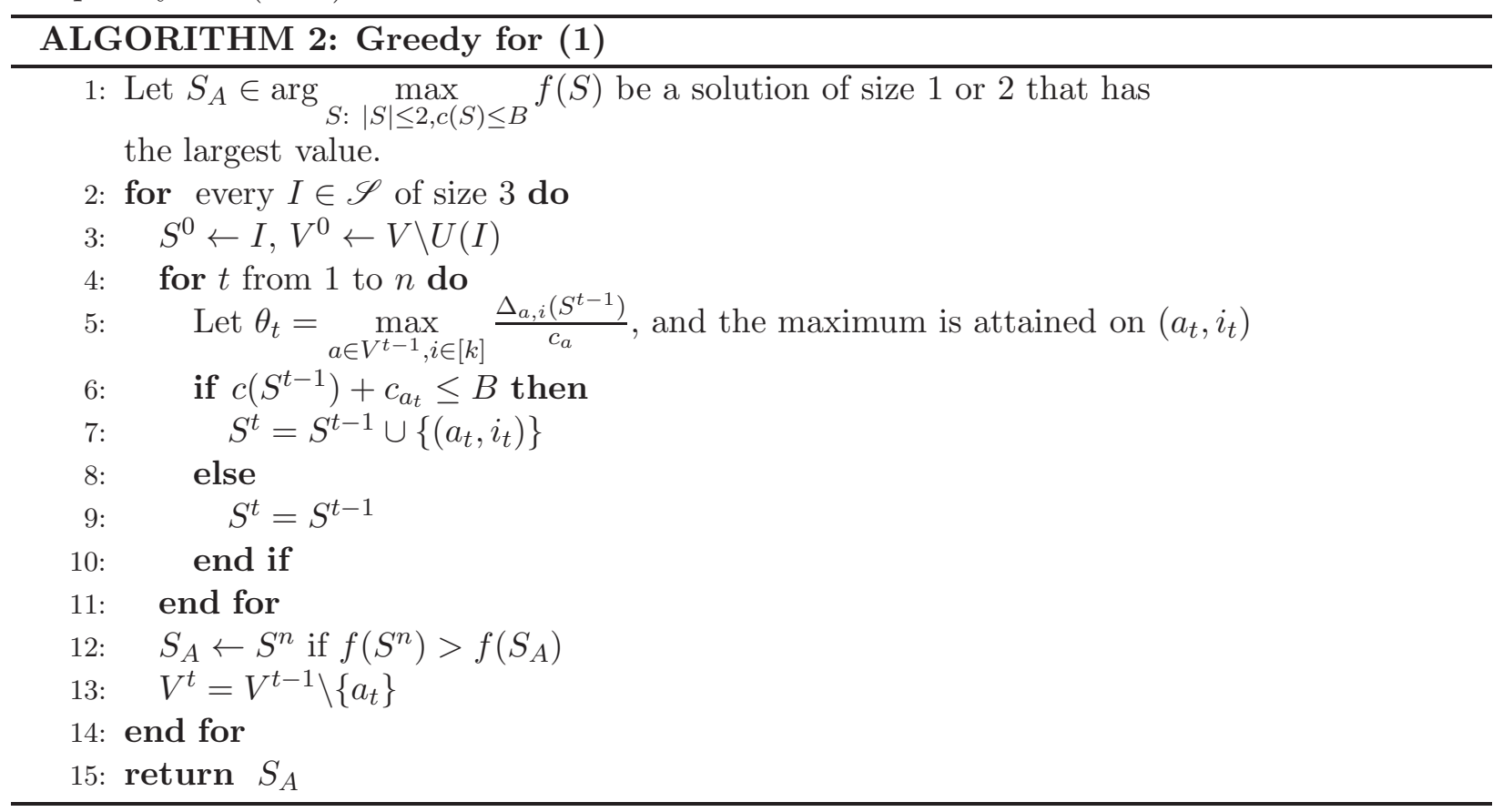

Theorem 3.1. For maximizing $f$ under a knapsack constraint, Algorithm 0 has an approximation ratio of $\frac{1}{2}-\frac{1}{2 e}$, and evaluates $f O\left(n^{5} k^{4}\right)$ times.

Proof. Let $T=\left\{\left(a_{1}^{*}, i_{1}^{*}\right), \ldots,\left(a_{|T|}^{*}, i_{|T|}^{*}\right)\right\}$ be an optimal solution. If $|T|=1$ or 2 , our algorithm must find it in Line 1 . So we only need to consider $|T| \geq 3$. We order the set $T$ so that for any $t=1, \ldots,|T|$,

$$
f\left(T^{t}\right)=\max _{(a, i) \in T \backslash T^{t-1}} f\left(T^{t-1} \cup\{(a, i)\}\right),
$$

where $T^{t}=\left\{\left(a_{1}^{*}, i_{1}^{*}\right), \ldots,\left(a_{t-1}^{*}, i_{t-1}^{*}\right)\right\}$, and $T^{0}=\emptyset$.

Let $Y=T^{3}$ be the set that consists of the first three items of $T$. For any item $\left(a_{j}^{*}, i_{j}^{*}\right) \in T$, $j \geq 4$, and set $Z \subseteq V \backslash\left\{a_{1}^{*}, a_{2}^{*}, a_{3}^{*}, a_{j}^{*}\right\} \times[k]$, we have

$$
\begin{aligned}
& f\left(Y \cup Z \cup\left\{\left(a_{j}^{*}, i_{j}^{*}\right)\right\}\right)-f(Y \cup Z) \leq f\left(T^{1}\right)-f(\emptyset) \leq f\left(T^{1}\right) ; \\
& f\left(Y \cup Z \cup\left\{\left(a_{j}^{*}, i_{j}^{*}\right)\right\}\right)-f(Y \cup Z) \leq f\left(T^{1} \cup\left\{\left(a_{j}^{*}, i_{j}^{*}\right)\right\}\right)-f\left(T^{1}\right) \leq f\left(T^{2}\right)-f\left(T^{1}\right) ; \\
& f\left(Y \cup Z \cup\left\{\left(a_{j}^{*}, i_{j}^{*}\right)\right\}\right)-f(Y \cup Z) \leq f\left(T^{2} \cup\left\{\left(a_{j}^{*}, i_{j}^{*}\right)\right\}\right)-f\left(T^{2}\right) \leq f\left(T^{3}\right)-f\left(T^{2}\right) .
\end{aligned}
$$

Summing up the above inequalities, we obtain

$$
f\left(Y \cup Z \cup\left\{\left(a_{j}^{*}, i_{j}^{*}\right)\right\}\right)-f(Y \cup Z) \leq f(Y) / 3 .
$$


From now on, we consider an iteration of the algorithm in which the set $Y$ was chosen at the beginning of the greedy procedure, i.e. $S^{0}=Y$. Define a function $g(S)=f(S)-f(Y)$ for all $S \supseteq Y$, which is also a monotone $k$-submodular function.

Let $t^{*}+1$ be the first step for which the algorithm does not add item $a_{t^{*}+1} \in U(T)$ to the set $U\left(S^{t^{*}}\right)$, i.e., $S^{t^{*}+1}=S^{t^{*}}$. We can further assume that $t^{*}+1$ is the first step $t$ for which $S^{t}=S^{t-1}$. This assumption is without loss of generality, because if it happens earlier for some $t^{\prime}<t^{*}+1$, then $a_{t^{\prime}} \notin U(T)$, and $a_{t^{\prime}}$ does not belong to the approximate solution we are interested in; therefore, excluding $a_{t^{\prime}}$ from the ground set $V$ does not change the analysis, the optimal solution $T$, and the approximate solution obtained in the iteration with $S^{0}=Y$.

Note that $Y \subseteq T \cap S^{t}$. Define $O P T_{g}\left(V^{\prime}\right)$ to be the optimal value of function $g$ over items $V^{\prime} \subseteq V$ without constraint. We greedily construct a set $\tilde{S} \in \mathscr{S}$ over items $U(T) \cup U\left(S^{t}\right)$ : starting with $Y \subseteq \tilde{S}$, consider every item in $U\left(S^{t} \backslash Y\right)$ in the same order as it is added to $U\left(S^{t}\right)$ in Algorithm 2. and then consider every item in $U(T) \backslash U\left(S^{t}\right)$ in an arbitrary order; when considering each item, assign the best index that brings the largest marginal gain. Clearly $S^{t} \subseteq \tilde{S}$, as the indices in $S^{t}$ are assigned greedily. For any $t=0, \ldots, t^{*}$, we have

$$
\begin{aligned}
g(T) & \leq O P T_{g}\left(U(T) \cup U\left(S^{t}\right)\right) \leq 2 \cdot g(\tilde{S}) \\
& \leq 2\left(g\left(S^{t}\right)+\sum_{(a, i) \in \tilde{S} \backslash S^{t}}\left(g\left(S^{t} \cup\{(a, i)\}\right)-g\left(S^{t}\right)\right)\right) \\
& =2\left(g\left(S^{t}\right)+\sum_{(a, i) \in \tilde{S} \backslash S^{t}}\left(f\left(S^{t} \cup\{(a, i)\}\right)-f\left(S^{t}\right)\right)\right) \\
& \leq 2\left(g\left(S^{t}\right)+(B-c(Y)) \theta_{t+1}\right) .
\end{aligned}
$$

The second inequality follows from the fact that $\tilde{S}$ is obtained greedily and Theorem 2.2. The third inequality is because of Lemma 2.1. The last inequality follows from $f\left(S^{t} \cup\{(a, i)\}\right)-f\left(S^{t}\right) \leq c_{a} \cdot \theta_{t+1}$ and $\sum_{(a, i) \in \tilde{S} \backslash S^{t}} c_{a} \leq B-c(Y)$.

Let $B_{t}=\sum_{\tau=1}^{t} c_{a_{\tau}}$ and $B_{0}=0$. Define $B^{\prime}=B_{t^{*}+1}$ and $B^{\prime \prime}=B-c(Y)$. By the definition of the item $a_{t^{*}+1}$, we have $B^{\prime}>B \geq B^{\prime \prime}$. For $j=1, \ldots, B^{\prime}$, we define $\rho_{j}=\theta_{t}$ if $j=B_{t-1}+1, \ldots, B_{t}$ (that is, $\rho_{1}=\cdots=\rho_{B_{1}}=\theta_{1}, \rho_{B_{1}+1}=\cdots=\rho_{B_{2}}=\theta_{2}, \ldots, \rho_{B_{t^{*}+1}}=\cdots=\rho_{B^{\prime}}=\theta_{t *+1}$ ). Using this definition, we obtain $g\left(S^{t}\right)=\sum_{\tau=1}^{t} c_{a_{\tau}} \theta_{\tau}=\sum_{j=1}^{B_{t}} \rho_{j}$ for $t=1, \ldots, t^{*}$ and $g\left(S^{t} \cup\left\{\left(a_{t^{*}+1}, i_{t^{*}+1}\right)\right\}\right)=$ $\sum_{\tau=1}^{t^{*}+1} c_{a_{\tau}} \theta_{\tau}=\sum_{j=1}^{B^{\prime}} \rho_{j}$. Then we have equalities

$$
\begin{aligned}
\min _{s=1, \ldots, B^{\prime}}\left\{\sum_{j=1}^{s-1} \rho_{j}+B^{\prime \prime} \rho_{s}\right\} & =\min _{t=0, \ldots, t^{*}}\left\{\sum_{j=1}^{B_{t}} \rho_{j}+B^{\prime \prime} \rho_{B_{t}+1}\right\} \\
& =\min _{t=0, \ldots, t^{*}}\left\{g\left(S^{t}\right)+B^{\prime \prime} \theta_{t+1}\right\} .
\end{aligned}
$$


Combining (4) with (3) and Lemma 2.3, we obtain

$$
\begin{aligned}
\frac{g\left(S^{t^{*}} \cup\left\{\left(a_{t^{*}+1}, i_{t^{*}+1}\right)\right\}\right)}{g(T)} & =\frac{\sum_{j=1}^{B^{\prime}} \rho_{j}}{g(T)} \\
& \geq \frac{\sum_{j=1}^{B^{\prime}} \rho_{j}}{2 \cdot \min _{s=1, \ldots, B^{\prime}}\left\{\sum_{j=1}^{s-1} \rho_{j}+B^{\prime \prime} \rho_{s}\right\}} \\
& \geq \frac{1}{2}\left(1-e^{-B^{\prime} / B^{\prime \prime}}\right)>\frac{1}{2}\left(1-e^{-1}\right) .
\end{aligned}
$$

Finally, combining (2) and (5), we obtain

$$
\begin{aligned}
f\left(S^{t^{*}}\right) & =f(Y)+g\left(S^{t^{*}}\right) \\
& =f(Y)+g\left(S^{t^{*}} \cup\left\{\left(a_{t^{*}+1}, i_{t^{*}+1}\right)\right\}\right)-g\left(S^{t^{*}} \cup\left\{\left(a_{t^{*}+1}, i_{t^{*}+1}\right)\right\}\right)+g\left(S^{t^{*}}\right) \\
& =f(Y)+g\left(S^{t^{*}} \cup\left\{\left(a_{t^{*}+1}, i_{t^{*}+1}\right)\right\}\right)-\left(f\left(S^{t^{*}} \cup\left\{\left(a_{t^{*}+1}, i_{t^{*}+1}\right)\right\}\right)-f\left(S^{t^{*}}\right)\right) \\
& \geq f(Y)+\frac{1}{2}\left(1-e^{-1}\right) g(T)-f(Y) / 3 \\
& \geq \frac{1}{2}\left(1-e^{-1}\right) f(T) .
\end{aligned}
$$

\section{References}

[1] Igor Gridchyn and Vladimir Kolmogorov. Potts model, parametric maxflow and k-submodular functions. In Proceedings of the IEEE International Conference on Computer Vision, pages 2320-2327, 2013.

[2] Hiroshi Hirai and Yuni Iwamasa. On k-submodular relaxation. SIAM Journal on Discrete Mathematics, 30(3):1726-1736, 2016.

[3] Anna Huber and Vladimir Kolmogorov. Towards minimizing k-submodular functions. In International Symposium on Combinatorial Optimization, pages 451-462. Springer, 2012.

[4] Satoru Iwata, Shin-ichi Tanigawa, and Yuichi Yoshida. Improved approximation algorithms for k-submodular function maximization. In Proceedings of the twenty-seventh annual ACM-SIAM symposium on Discrete algorithms, pages 404-413. SIAM, 2016.

[5] Lan Nguyen and My T Thai. Streaming k-submodular maximization under noise subject to size constraint. In International Conference on Machine Learning (ICML), pages 7338-7347. PMLR, 2020.

[6] Naoto Ohsaka and Yuichi Yoshida. Monotone k-submodular function maximization with size constraints. In Proceedings of the 28th International Conference on Neural Information Processing Systems (NeurIPS), volume 1, pages 694-702, 2015.

[7] Hiroki Oshima. Improved randomized algorithm for k-submodular function maximization. SIAM Journal on Discrete Mathematics, 35(1):1-22, 2021. 
[8] Shinsaku Sakaue. On maximizing a monotone k-submodular function subject to a matroid constraint. Discrete Optimization, 23:105-113, 2017.

[9] Tasuku Soma. No-regret algorithms for online $k$-submodular maximization. In The 22nd International Conference on Artificial Intelligence and Statistics, pages 1205-1214. PMLR, 2019 .

[10] Maxim Sviridenko. A note on maximizing a submodular set function subject to a knapsack constraint. Operations Research Letters, 32(1):41-43, 2004.

[11] Justin Ward and Stanislav Živnỳ. Maximizing k-submodular functions and beyond. ACM Transactions on Algorithms (TALG), 12(4):1-26, 2016.

[12] Laurence A Wolsey. Maximising real-valued submodular functions: Primal and dual heuristics for location problems. Mathematics of Operations Research, 7(3):410-425, 1982. 